\title{
U-SMART : SMALL APERTURE ROBOTIC TELESCOPES FOR UNIVERSITIES
}

\author{
P. Gebhardt ${ }^{1}$, A. Schrimpf ${ }^{1}$, C. Dersch ${ }^{1}$, M. Spasovic ${ }^{1}$, L. Bringmann ${ }^{1}$, H. P. Singh ${ }^{2}$, R. Gupta $^{3}$, \\ and S. M. Kanbur ${ }^{4}$
}

\begin{abstract}
A group of universities have come together with the aim of designing and developing small aperture robotic telescopes (SmART) for use by students to observe variable stars and transient follow-ups. The group is deliberating on the components of the robotic system e.g. the telescope, the mount, the back-end camera, control software etc and their integration keeping in mind the scientific objectives. The Marburg group is studying variable stars using photometric and spectroscopic observations in a small local observatory and via analysis of photo plate archival data mainly from Sonneberg observatory (Thuringa, Germany). Our goal is to setup a small, affordable observatory at Marburg University with full remote access to all components, including a spectrometer. Among others, the future measurements can be follow-ups, of variable stars, exoplanet search and spectroscopic measurements, e.g. in cooperation with the BRITE-constellation. We would like to invite other universities to join the project and cooperate in setting up a University network of small aperture robotic telescopes (U-SmART) around the globe.
\end{abstract}

\section{RESUMEN}

Un conjunto de universidades han acordado diseñar y desarrollar telescopios robóticos de pequeño diámetro (SmART) para ser usados por estudiantes de modo que puedan observar estrellas variables y realizar seguimientos ópticos de fenónemos transitorios. El grupo está reconsiderando los componentes del sistema robótico, i.e. el telescopio, la montura, la cámara, el software de control, etc. y su integración teniendo presente los objetivos científicos. El grupo de Marburg está estudiando las estrellas variables usando las observaciones fotométricas y espectroscópicas de un pequeño observatorio local y analizando las placas fotográficas de archivo obtenidas en el Observatorio de Sonneberg (Turingia, Alemania). Nuestro objetivo es instalar un observatorio modesto en la Universidad de Marburg con acceso remoto completo a todos sus componentes, incluyendo un espectrómetro. Entre otras, las medidas futuras pueden ser de seguimiento, de estrellas variables, de búsqueda de exoplanetas y de toma de espectros, por ejemplo cooperando con la constelación de nanosatélites BRITE. Aprovechamos para invitar a otras universidades a unirse al proyecto y cooperar desplegando una red de telescopios universitarios de pequeño diámetro (U-SmART) en todo el planeta.

Key Words: spectroscopy techniques: miscellaneous - telescopes

\section{MAIN IDEA}

The main idea is the construction of a global network of small aperture university telescopes, all accessible via internet to students and members of the participating institutions. A telescope connection opens up the possibility of follow-ups and continuous measurements of transits in young star systems, novae, variable stars, etc. Figure 1 gives an example light curve of the dwarf nova RX And with data taken at Marburg and from the AAVSO. We pro-

\footnotetext{
${ }^{1}$ Philipps-University of Marburg, History of Astronomy and Observing Astronomy, Renthof 7b, 35039 Marburg, Germany (patrick.gebhardt, andreas.schrimpf@physik.unimarburg.de).

${ }^{2}$ University of Delhi, India.

${ }^{3}$ University of Pune, India.

${ }^{4}$ State University of New York, Oswego, USA.
}

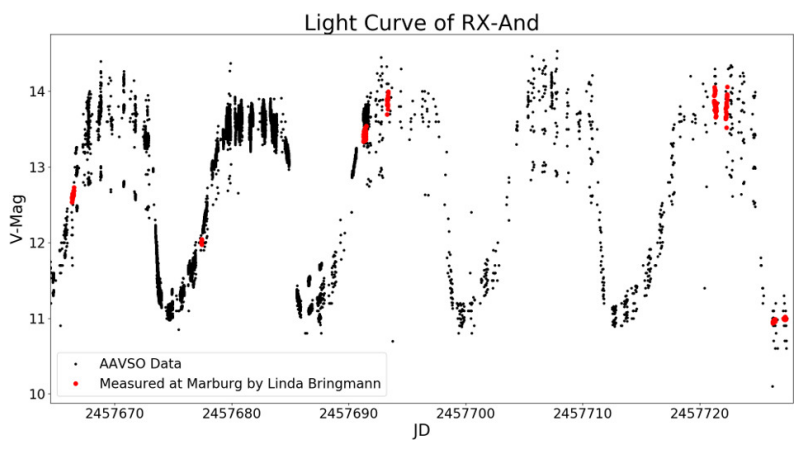

Fig. 1. Light curve of RX And, data taken at Marburg (red) in comparison with AAVSO data (black). The Marburg data were measured in the V band with an 8" Schmidt-Cassegrain and an Atik 383L+ camera. 


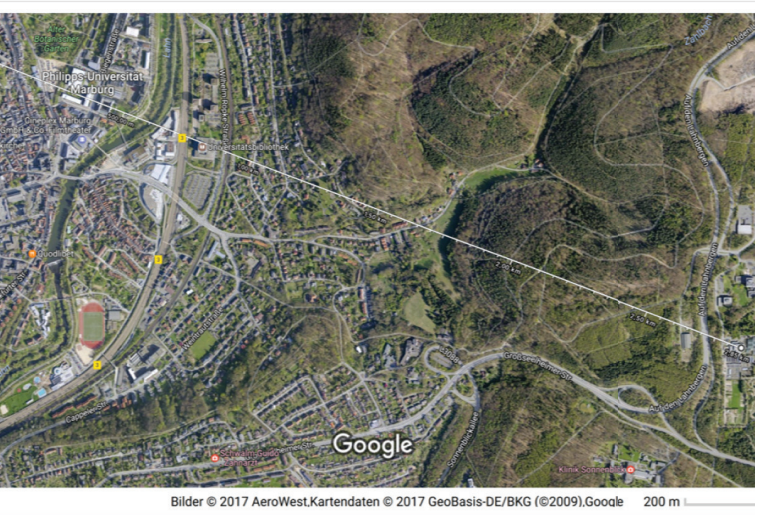

Fig. 2. Finding a new location at Lahnberge, Marburg

pose to create a network of affordable instruments using open-source software. Any university observatory that fulfills the minimum local demands may join the network.

\section{REALIZATION}

\subsection{Marburg Observatory}

The recent setup at Marburg consists of a Celestron CGE pro mount, an 8" f/3.6 astrograph, an 8" f/10 Schmidt-Cassegrain, an Atik 383L+ camera, an Atik 314 camera, different filter sets, as well as a LHIRES III spectograph.

Our setup - except of the spectrograph - is controlled using the INDI library (Downey et al. 2007) on a small local computer and we successfully run remote measurements within the university network. For our measurements we use KStars (remote client to control measurements and scheduling) on Linux systems.

The current location of our setup at Physics Department has poor light and measurement conditions. Looking for a better site we performed several observation at the botanical garden of the university about $3 \mathrm{~km}$ from the center of the city (see Figure 2). The results are very promising: less light pollution and better $\mathrm{S} / \mathrm{N}$ ratio.

\subsection{Other Observatories}

Several sites are considering to be included in the U-SmART network (Gupta et al. 2015). H. P. Singh is in planning a new telescope at Hanle Observatory, Ladakh, India. Ladakh is one of the darkest places in the world. R. Gupta intends to join with an observatory at Puna, India, and S. M. Kanbur is planing to include the setup at Oswego, NY, USA, into the new telescope network. Scientists interested in helping to setup and/or to join the network may contact one of the authors.

\section{INDI (INSTRUMENT NEUTRAL DISTRIBUTED INTERFACE) LIBRARY}

For U-SmART we propose to use the INDI library for observatory control because of its distinct features (see Figure 3):

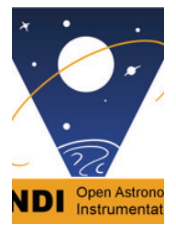

- Distributed control: With INDI server/client architecture, you can communicate with devices either locally or remotely transparently.

- Open source software: INDI is released under the GNU Lesser General Public License (LGPL $\mathrm{v} 2+$ ), you can use and modify the source code to fit your needs in any environment.

- Cross Platform: Currently Linux, BSD, and OSX supported the INDI library. Windows support is under progress.

- Wide range of support: INDI supports many astronomical devices and furthermore, INDI clients include popular astronomy software suites such as KStars, Xephem and SkyCharts.

- XML based: INDI is small and easy is parse. The INDI protocol can be nested within other XML elements such as RTML to add constraints for automatic scheduling and execution.

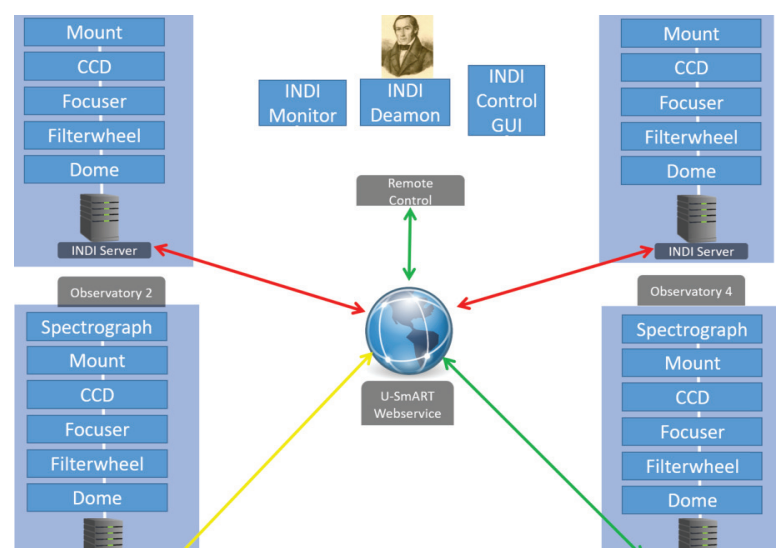

Fig. 3. The possible structure of U-SmART. (Historical observer C.L. Gerling in 1841). 


\section{OUTLOOK}

\subsection{Web Service}

So far only single user clients exist for INDI library, e.g. KStars. It is necessary to setup up a multi user environment, a local database, a scheduler and a web service to get remote access to the observatories.

Some observatories use RTS2 (Kubánek et al. 2004) for control and scheduling and GLORIA (Castro-Tirado et al. 2014) for remote access. However, INDI seems to be attracting a wider community. We intend to get in contact with the RTS2 and GLORIA developers to discuss and realize an integration of INDI for hardware control into RTS2 and GLORIA.

\subsection{Photometric Software Pipeline}

Currently a photometric python based software pipeline is under development. This software pipeline includes astronomical data reduction and differential photometry and uses astropy (Robitaille et al. 2013), astrometry.net (Lang et al. 2010). It will be released as an open source software package.

\subsection{Possible Setup of the New Marburg Observatory}

We are planing to setup up a new observatory at Lahnberge, Marburg. Table 1 shows a possible components of the observatory, motivated by the APASS setup (Smith et al. 2010).
TABLE 1

\section{POSSIBLE SETUP OF THE NEW OBSERVATORY AT THE LAHNBERGE AT MARBURG}

\begin{tabular}{ll}
\hline Devices & Name \\
\hline Dome & Astroshell \\
Mount & Paramount MX+ \\
Telescope & ASA Teleskop \\
CCD & Apogee Aspen \\
Guiding camera & Apogee \\
Filterwheel & Apogee \\
Filters & SDSS \\
Spectroscope & Baches, at f/10-optics \\
Weather station & MBox Meteostation \\
All sky camera & SkyEye \\
\hline
\end{tabular}

\section{REFERENCES}

Castro-Tirado, A. J. et al. 2014, RMxAC, 45, 104

Downey, E. C. 2007, INDI: Instrument-NeutralDistributed-Interface, url http://indilib.org

Gupta, R., Singh, H. P., Kanbur, S. M., Schrimpf, A., \& Dersch, C. 2015, PKAS, 30, 683

Kubánek, P., Jelínek, M., Nekola, M., Topinka, M., Štrobl, J., et al. 2004, AIPC 727, 753

Lang, D., Hogg, D. W., Mierle, K., Blaton, M., \& Roweis, S. 2010, AJ, 139, 17282

Robitaille, T. P., Tollerud, E. J., Greenfield, P., Droettboom, M., Bray, E., et al. 2013, A\&A, 558, 33

Smith, T. C., Henden, A., \& Terrell, D. 2010, SASS, 29, 45 